\title{
Commentary An early peak of relapse after surgery for breast cancer
} Anthony Howell

\author{
CRUK Department of Medical Oncology, Christie Hospital, University of Manchester, Manchester, UK \\ Corresponding author: Anthony Howell, Anthony.Howell@christie-tr.nwest.nhs.uk \\ Published: 11 October 2004 \\ Breast Cancer Res 2004, 6:255-257 (DOI 10.1186/bcr946) \\ (C) 2004 BioMed Central Ltd \\ See related research article by Demicheli et al., http://breast-cancer-research.com/content/6/6/R689
}

\begin{abstract}
There is great interest among oncologists concerning what we might learn by examining the pattern of relapse after breast cancer surgery. What you see depends upon how hard you look. Up to now, investigators have examined the hazard ratio for relapse every 6-12 months. In a research paper, published in this issue of Breast Cancer Research, the Milan group have looked at the hazard ratio every three months and have found, for the first time, a distinct, very early peak of relapse in a group of premenopausal, node-positive patients not given chemotherapy or hormone therapy. What is now needed is for other groups to repeat this observation and, if found, to examine the characteristics of the tumours producing this phenomenon in order to develop hypotheses about its cause and possible treatments.
\end{abstract}

Keywords: angiogenesis, peak of relapse, therapy, wounding

\section{Introduction}

The recent research paper by the Milan group reports the time to recurrence after removal of a primary breast tumour in a group of 1173 patients entered into clinical trials in Milan between 1964 and 1980 [1]. All patients were treated by radical or modified radical mastectomy but they did not receive postoperative radiotherapy or chemotherapy. Previous analyses of this patient series estimating the hazard ratio for relapse at 6-12 month intervals reported peaks of relapse at 18 months and 60 months and then a tapered plateau like tail of relapse extending up to 15 years [2]. The early peak was more pronounced in patients with node-positive tumours that were more than $2 \mathrm{~cm}$ in diameter. No differences in the curves were seen for local and distant relapse or for pre- and postmenopausal patients. At the same time Saphner and colleagues reported similar findings [3], with the additional observation that the early peak was significantly higher in oestrogen receptor (ER) negative tumours. No ER data are available for the Milan series.

In the current paper by the Milan group, the aim of the authors was to further interrogate the kinetics of the first peak. Hazard rates were calculated at three month intervals and the authors report in detail 368 patients with a local (95) or distant (273) relapse within the first four years after surgery. The analysis demonstrates, for the first time, a double peak of early relapse with maximum at 8-10 months and 28-30 months for premenopausal patients, but a wide peak with a maximum at 18-24 months for postmenopausal patients. When divided into node-positive and node-negative, the double pattern was seen only in node-positive premenopausal patients: it was more pronounced in patients with more than three nodes involved, but was unrelated to tumour size.

This analysis therefore confirms a previous one in the same group of patients, which showed that $27 \%$ of all distant relapses of premenopausal node-positive patients occurred within the first ten months, if not treated with adjuvant therapy [4]. The first question to ask is whether the differences between pre- and postmenopausal patients are real. The authors did not assess the differences between the curves statistically, but by eye there does appear to be a definite early peak in the premenopausal group. Given the relatively small numbers 
of patients involved, the analysis needs repeating in another large series. An early peak was not seen in the Saphner series but the hazard ratios were only examined annually.

\section{Why is there an early peak of recurrence?}

If we assume there is a peak of recurrence at 8-10 months we have to explain why there is a peak at all, why so soon after surgery and why only in premenopausal women. There was no break down of the data to tell us whether the phenomenon was associated with younger or older premenopausal women. This would be of interest because of the particularly poor prognosis of very young breast cancer patients.

The authors' explanation for the early peak of recurrence is that surgery triggers the release of micrometastases from a dormant state either by stimulating the release of angiogenic factors or inhibiting angiogenic inhibitors. In previous publications they have also hypothesised that growth factors released during the wounding of surgery may also stimulate tumour growth [5]. It is important to emphasise the great heterogeneity of breast tumours and the variability in times of relapse. Tumours that relapse after some years tend to be steroid hormone receptor (HR) positive whereas those that relapse early tend to be HR negative and HER1/HER2 positive. In late relapses micrometastases may well be dormant for months or years and then begin to grow. This is best seen in local relapse where clinical examination may find nothing on several occasions, and on the next examination a small mass is found (or more often, detected by the patient) [6]. This suggests dormancy followed by growth. Whether growth is initiated by an acquired ability of tumour cells to induce angiogenesis is not clear, but this is a widely held hypothesis. Micrometastases in axillary lymph nodes have few blood vessels, whereas larger nodal metastases are well vascularised suggesting that there may be an angiogenetic switch [7]. However, in animal models where tumour cells are injected into, for example, the portal system, single cells can be found in the liver many months afterwards. These cells can form tumours when they are removed from the liver and injected into mammary fat pads; therefore the liver environment must be keeping the cells dormant in some way [8]. This is unlikely to be related to angiogenesis since the tumour cells are angiogenic when transferred to the fat pad, and recent evidence suggests that metastatic breast tumours can use existing blood vessels in the liver [9].

At the other end of the heterogeneity scale are tumours that relapse early (the subject of the recent research paper). Although we do not have any histological or immunochemical details of the tumours used by the Milan group, data from other studies indicate that the tumours are likely to be not only HR negative and HER1/2 positive, vascular invasion [10-12]. The fact that lymph node metastases are often large and vascularised at diagnosis in these cases indicates that we need not necessarily involve an angiogenic switch (as the authors suggest) since at the time of surgery not only the primary tumour, but also detectable metastases are well vascularised. Nor need we necessarily invoke dormancy, since this group of tumours have already shown their ability to grow and vascularise in axillary lymph nodes before operation. The early peak could simply represent a subgroup of tumours with similar growth characteristics uninterrupted by surgery. It is of interest that tumour cells can be detected in peripheral blood two to three weeks after surgery in poor prognosis groups, as this suggests release from existing subclinical metastases [12].

\section{Angiogenesis inhibition}

The hypothesis that the primary tumour somehow affects the growth of metastases is compelling. Large numbers of animal experiments show that removal of the primary tumour is associated with increased proliferation of smaller tumours, whether they are metastases or small inoculae of the same tumour type. Holmgren and colleagues [13] subcutaneously inoculated mice with Lewis lung carcinoma or the T241 sarcoma and showed that dormant micrometastases developed in the lungs. The inoculae were removed when they reached a volume of $1.5-2.0 \mathrm{~cm}$, which triggered the growth of the lung micrometastases. It was further shown that treatment of the mice with an angiogenesis inhibitor (TNP-470) prevented the growth of lung metastases suggesting that release from dormancy is via an angiogenic switch. However, Fisher and colleagues [14] demonstrated that increased growth of small transplanted tumours in one flank, after removal of a large 'primary' tumour in the other, was associated with factors in serum that could not only stimulate tumour growth in vivo in other animals, but also of the same tumour cells in vitro. The latter experiment suggests that the factor or factors were not angiogenic. Proliferative factors were present in the serum within hours of the operation, but wounding did not appear to explain this result, since a sham operation (removal of the hind limb) did not result in tumour growth.

Aside from Fisher's experiment, other data support the wounding hypothesis. Recently, Tagliabue and colleagues demonstrated that removal of part of a primary human mammary tumour resulted in increased growth rates of residual primary tumour (incomplete excision) but only in tumours that were HER-2 positive. Wound drainage fluid and post surgical serum samples stimulated growth of HER-2 over-expressing cells, which were abolished by trastuzumabab [15]. These experiments are important because measurements were made on fluids from wounds, which may not be reflected in blood measurements. Hombrey and colleagues [16] 
demonstrate very high concentrations of vascular endothelial growth factor (VEGF) in wound fluid but small changes in peripheral blood VEGF levels after surgery for breast cancer. It is possible that the early peak of relapse is seen only in premenopausal women because the breast is primed to produce proliferative factors by high endogenous steroid hormones.

\section{Conclusions}

The data outlined above suggest that the explanation for the peak of relapse seen at 8-10 months in the Milan series is related to a group of tumours that other studies suggest are inherently highly vascularised and metastatic. The presence of lymph node metastases at diagnosis suggests the capacity for angiogenesis is present in the cells, and there are probably metastases in other sites which are also quite large, given the finding of tumour cells in the peripheral blood after a period of two to three weeks. Whether there is an increased growth of metastases at the time of surgery, either due to growth stimulators or removal of inhibitors produced by the tumour or by wounding, is suggested by the peak of relapse, but this remains to be clearly demonstrated in humans. The limited experimental data we have indicates a need for further clinical studies to define the potential regulators involved. There is also a need to assess whether treatment across the surgical period with HER1/2 inhibitors, for example, will help improve the outcome of patients with poor prognosis tumours that do not respond well to conventional therapies.

\section{Competing interests}

The author(s) declare that they have no competing interests.

\section{References}

1. Demicheli R, Bonadonna G, Hrushesky WJM, Retsky MW, Valagussa $P$ : Menopausal status dependence of the timing of breast cancer recurrence following primary tumour surgical removal. Breast Cancer Res 2004, 6:R689-R696.

2. Demicheli R, Abbattista A, Miceli R, Valagussa P, Bonadonna G: Time distribution of the recurrence risk for breast cancer patients undergoing mastectomy: further support about the concept of tumor dormancy. Breast Cancer Res Treat 1996, 41:177-185.

3. Saphner T, Tormey DC, Gray R: Annual hazard rates of recurrence for breast cancer after primary therapy. J Clin Oncol 1996, 14: 2738-2746.

4. Retsky M, Demicheli R, Hrushesky W: Premenopausal status accelerates relapse in node positive breast cancer: hypothesis links angiogenesis, screening controversy. Breast Cancer Res Treat 2001, 65:217-224.

5. Demicheli R, Valagussa $\mathrm{P}$, Bonadonna G: Does surgery modify growth kinetics of breast cancer micrometastases? $\mathrm{Br} J$ Cancer 2001, 85:490-492.

6. Demicheli R, Terenziani M, Bonadonna G: Estimate of tumor growth time for breast cancer local recurrences: rapid growth after wake-up? Breast Cancer Res Treat 1998, 51:133-137.

7. Klauber-DeMore N, Kimberly J, Van Zee KJ, Linkov I, Borgen PI, Gerald WL: Biological behaviour of human breast cancer micrometastases. Clin Cancer Res 2001,7:2434-2439.

8. Naumov GN, MacDonald IC, Weinmeister PM, Kerkvliet N, Nadkarni KV, Wilson SM, Morris VL, Groom AC, Chambers AF: Persistence of solitary mammary carcinoma cells in a secondary site: a possible contributor to dormancy. Cancer Res 2002, 62: 2162-2168.
9. Stessels F, Van den Eynden G, Van der Auwera I, Salgado R, Van den Heuvel E, Harris AL, Jackson DG, Colpaert CG, Van Marck EA, Dirix LY, Vermeulen PB: Breast adenocarcinoma liver metastases, in contrast to colorectal cancer liver metastases, display a non-angiogenic growth pattern that preserves the stroma and lacks hypoxia. Br J Cancer 2004,90:1429-1436

10. Faneyte IF, Peterse JL, van Tinteren H, Pronk C,deVries EGE, Rodenhuis S, van de Vijver : Predicting early failure after adjuvant chemotherapy in high risk breast cancer patients with extensive lymph node involvement. Clin Cancer Res 2004, 10: 4457-4463.

11. Slamon DJ, Clark GM, Wong SG, Levin WJ, Ullrich A, McGuire WL: Human breast cancer: correlation of relapse and survival with amplification of the HER-2/neu oncogene. Science 1987, 235:177-182.

12. Nielson TO, Hsu FD, Jensen $K$, Cheang M, Karaca G, Hu Z, Hernandez-Boussard T, Livasy C, Cowan D, Dressler L, Akslen LA, Ragaz J, Gown AM, Blake Gilks C, van de Rijn M, Perou CM: Immunohistochemical and clinical characterisation of the basal-like subtype of invasive breast carcinoma. Clin Cancer Res 2004, 10:5367-5374.

13. Holmgren L, O'Reilly MS, Folkham J: Dormancy of micrometastases: balanced proliferation and apoptosis in the presence of angiogenesis suppression. Nat Medicine 1995, 1:149-153.

14. Fisher B, Gunduz N, Coyle J, Rudock C, Saffer E: Presence of a growth-stimulating factor in serum following primary tumor removal in mice. Cancer Res 1989, 49:1996-2001.

15. Tagliabue E, Agresti R, Carcangiu ML, Ghirelli C, Morelli D,Campiglio M, Martel M, Giovanazzi R, Greco M, Balsari A, Menard S: Role of HER2 in wound-induced breast carcinoma proliferation. Mechanisms of disease 2003, 362:527-533.

16. Hombrey E, Han C, Roberts A, McGrouther DA, Harris AL: The relationship of human wound vascular endothelial growth factor (VEGF) after breast cancer surgery to circulating VEGF and angiogenesis. Clin Cancer Res 2003, 9:4332-4339. 